\title{
USE OF NEAR INFRARED SPECTROSCOPY TO IDENTIFY TRAUMATIC INTRACRANIAL HEMATOMAS
}

\author{
Claudia S. Robertson, ${ }^{\dagger}$ Shankar P. Gopinath, ${ }^{\dagger}$ and Britton Chance* \\ ${ }^{\dagger}$ Baylor College of Medicine, Department of Neurosurgery, One Baylor Plaza, Houston, Texas 77030; \\ *University of Pennsylvania, Department of Biophysics and Biochemistry, Philadelphia, \\ Pennsylvania 19104 \\ (Paper JBO/NIR-16 received July 15, 1996; revised manuscript received Nov. 8, 1996; accepted for publication Dec. 2, 1996.)
}

\begin{abstract}
Delayed intracranial hematomas are an important treatable cause of secondary brain injury in patients with head trauma. Early identification and treatment of these mass lesions, which appear or enlarge after the initial CT scan, may improve neurological outcome. Serial examinations using near-infrared spectroscopy (NIRS) to detect the development of delayed hematomas were performed in 305 patients. The difference in optical density $(\Delta \mathrm{OD})$ at $760 \mathrm{~nm}$ between the normal and the hematoma side was measured on admission and then serially during the first 3 to 5 days after injury. On admission, the $\Delta$ OD was highly predictive of the initial findings on CT scan. Patients with an epidural, subdural, or intracerebral hematoma had significantly greater $\Delta \mathrm{OD}$ in the involved brain region than patients with diffuse brain injury. For the extracerebral hematomas, the $\Delta \mathrm{OD}$ was significantly related to the size of the hematoma $\left(r^{2}=0.55\right.$ and 0.77 for subdural and epidural hematomas, respectively). Fifty-nine (19\%) of the patients developed some type of late hematoma: there was an intracerebral hematoma in 29 patients, an extracerebral hematoma in 7 patients, and a postoperative hematoma in 23 patients. Thirty-three of the late hematomas were large enough to require surgical evacuation. The hematomas appeared between 2 and $72 \mathrm{~h}$ after admission. In 55 of the 59 patients, an increase in the $\Delta \mathrm{OD}$ to $>0.10$ occurred prior to an increase in intracranial pressure or a change in the neurological examination. Early diagnosis using NIRS may allow early treatment and reduce secondary injury caused by delayed hematomas. () 1997 Society of Photo-Optical Instrumentation Engineers.
\end{abstract}

Keywords near infrared spectroscopy; intracranial hematomas; CT scan.

\section{INTRODUCTION}

Intracranial hematomas are a treatable cause of secondary injury if identified early, but can cause significant disability or death if not promptly recognized and treated. They occur as the primary injury in $40 \%$ of patients with severe head injury. Recurrent hematomas, postoperative epidural hematomas, and delayed traumatic intracerebral hematomas develop in up to $23 \%$ of patients with severe head injury. ${ }^{1-10}$ Mortality rates and the incidence of a poor neurological recovery are significantly increased in patients who develop delayed traumatic intracranial hematomas. ${ }^{2,511,12}$ Early identification, prior to neurological deterioration, is the key to successful surgical treatment.

Serial CT scans are the most reliable method for detecting a delayed hematoma. However, CT scans require that patients, many of whom are critically ill, be taken out of the intensive care unit, and the yield is relatively low if serial scans are obtained in

Address all correspondence to Claudia S. Robertson. E-mail: claudiar@bcm.tmc.edu all patients. Some clinical monitoring technique for accurate selection of patients requiring follow-up CT scanning would improve the yield. Current clinical monitoring techniques, which include intracranial pressure (ICP) and monitoring and following the neurological status with Glasgow coma scores (GCS), are not ideal for detecting delayed hematomas. Patients with delayed hematomas may appear to be relatively normal, only to undergo sudden neurologic deterioration, ${ }^{5}$ or may not exhibit a change in their neurologic examination. $2,12,13$ Intracranial pressure may be normal in up to $20 \%$ of patients harboring delayed hematomas that require surgery. ${ }^{2,14,15}$

The purpose of these studies was to evaluate near-infrared spectroscopy (NIRS) as a bedside method for detecting intracranial hematomas that are present on admission or that develop during the first few days after admission for a traumatic head injury. 


\section{METHODS AND MATERIALS}

\subsection{PATIENT CHARACTERISTICS}

Three hundred and five patients with head injury were studied with NIRS examinations in the emergency room and serially in the intensive care unit. Patients with massive scalp lacerations, avulsions, and hematomas were excluded from the study. Four patients were excluded for this reason.

The age of the patients ranged from 4 months to 101 years. Fifty patients were female and 255 were male. One hundred sixty patients had a severe head injury (Glasgow coma score 3 to 8) and 145 had a moderate or mild head injury (Glasgow coma score 9 to 15).

All patients were evaluated with an initial CT scan and were followed with serial neurological examinations. Patients with Glasgow coma scores $\leqslant 8$ also had intracranial pressure monitoring. A repeat CT scan was also obtained after the occurrence of neurological deterioration, increasing ICP, or suggestion of an intracranial hematoma by NIRS examination. Indications for surgery were a midline shift greater than $5 \mathrm{~mm}$, intracranial hypertension, or neurological deterioration.

\subsection{METHOD OF NIRS EXAMINATION}

The principle used in identifying intracranial hematomas with NIRS is that extravascular blood absorbs NIR light more than normal brain tissue since there is a greater concentration of hemoglobin in the acute hematoma. Therefore, the absorbance of NIR light would be greater (and therefore the reflected light less) on the side of the brain containing a hematoma than on the uninjured side.

A dual wavelength reflectance spectrometer was used (RunMan, NIM, Inc., Philadelphia). This monitor is small $(6.5 \times 4.5 \times 2$ inches), battery operated, and can be easily transported into the emergency room or intensive care unit. The probe consists of two small incandescent bulbs placed 3.5 to $4.0 \mathrm{~cm}$ on either side of a 760- and 850-nm photodetector. The 3.5-cm separation was used in the first 46 patients, and the $4.0-\mathrm{cm}$ separation was used in the remainder of the patients. Leakage of the light is minimized by the presence of rubber dams between the light emitter and detectors, and around the circumference of the probe.

The procedure for an NIRS examination took less than $10 \mathrm{~min}$. The NIRS probe was placed successively in the frontal, temporal, parietal, and occipital areas of the head and, after equilibration of the reading, the intensity of reflected light at $760 \mathrm{~nm}$ was recorded and corresponding areas compared. Areas of the head with scalp hematomas, which were obvious on inspection, were specifically avoided. The difference in optical density $(\Delta O D)$ in the different areas was calculated from the following formula:

$$
\Delta O D=\log _{10}\left(\frac{I_{L}}{I_{R}}\right),
$$

where $I_{L}$ is the intensity of reflected light on the left side and $I_{R}$ is the intensity of reflected light on the right side.

By this definition, normal $\triangle O D$ was \pm 0.02 ; increasing absorbance (indicating increasing blood) on the left was indicated by a negative $\Delta O D$, and increasing absorbance (indicating increasing blood) on the right was indicated by a positive $\Delta O D$.

An NIRS examination was obtained in the emergency room at the time of the admission CT scan, and then serial measurements were obtained during the hospital course, along with follow-up CT scans. With each examination the $\triangle O D$ for each of the four brain regions was recorded, and the $\triangle O D_{\max }$, defined as the greatest absolute value for $\triangle O D$ among the various regions examined, was recorded.

\subsection{STATISTICAL ANALYSIS}

Data are expressed as the median and range. Data in Figure 1 are displayed as box plots. In these graphs, the horizontal line through the box marks the median value. The top and bottom of the box mark the $75^{\text {th }}$ and $25^{\text {th }}$ percentiles, respectively. The top and bottom error bars mark the $90^{\text {th }}$ and $10^{\text {th }}$ percentiles, respectively. Circles indicate any outlying points.

Differences in median values were compared by Kruskal-Wallis analysis of variance and Dunn's method when multiple comparisons were made. The $\Delta O D_{\max }$ was compared against the thickness of the hematoma on a CT scan by nonlinear regression analysis. Proportions were compared by the chisquare test. A $P$ value of less than 0.05 was considered significant.

\section{RESULTS}

\subsection{NIRS IDENTIFICATION OF INTRACRANIAL HEMATOMAS IN THE EMERGENCY ROOM}

The ability of NIRS to identify and localize intracranial hematomas in the emergency room was evaluated in the 305 patients. One hundred ninetyone of the patients had an intracranial hematoma on admission to the hospital, and 114 had an initial diagnosis of diffuse brain injury. The values for the $\Delta O D_{\max }$ on the initial NIRS examination in the 305 patients are summarized in Table 1.

The median $\triangle O D_{\max }$ for the 114 patients with diffuse brain injury was 0.03 (range 0 to 0.05 ), which was not significantly different from that observed in a group of 10 uninjured patients reported previously. ${ }^{16}$ In contrast, the 191 patients with intracranial hematomas had a median $\triangle O D_{\max }$ of 0.78 (range 0.03 to 1.82 ), which was significantly greater than both the uninjured patients and the pa- 


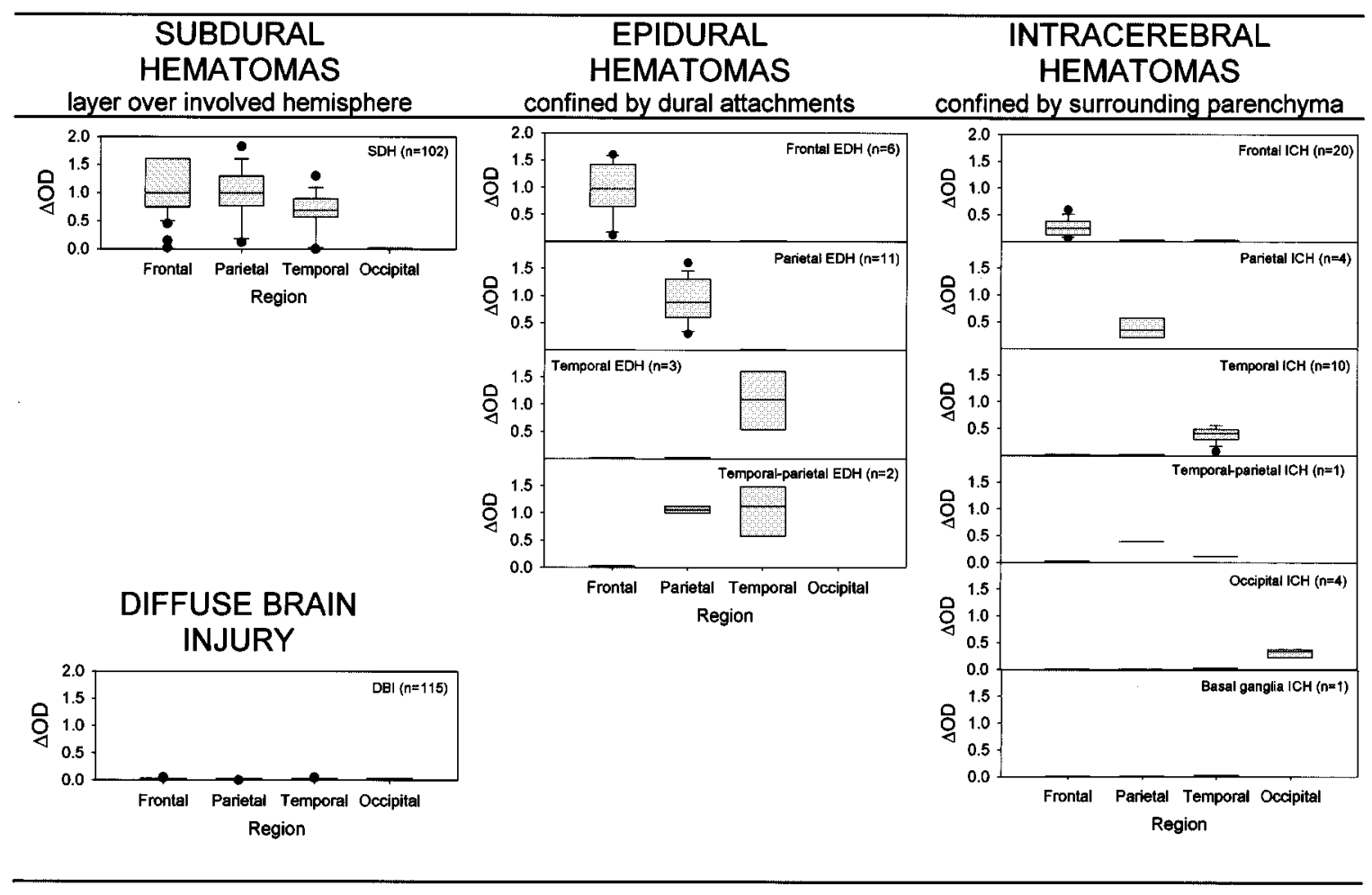

Fig. 1 The $\Delta O D_{\max }$ for the extracerebral hematomas was significantly related to the size of the hematoma.

tients with diffuse brain injury $(p<0.001)$. The $\Delta O D_{\max }$ was highest in the patients with the extracerebral types of hematomas, epidural and subdural, than with the contusions and intracerebral hematomas, and the $\triangle O D_{\max }$ for each of the three types of hematomas was significantly greater than the patients with diffuse brain injury $(p<0.001)$. Within each hematoma type, there was considerable variation in the $\triangle O D_{\max }$; however the differences in the size of the individual hematomas accounted for most of this variation. As shown in Figure 2, the $\Delta O D_{\max }$ of the extracerebral hematomas was sig- nificantly related to the thickness of the hematoma on the initial CT scan.

The type of hematoma was suggested by the degree of reduction in reflected light intensity (Table 1 ) and by the distribution of the changes in reflected light intensity (Figure 1). An $\Delta O D_{\max }$ of $>0.6$ was almost always an extracerebral hematoma (epidural or subdural). An $\Delta O D_{\max }$ value $>0.6$ occurred in only one patient with an intracerebral hematoma. $\Delta O D_{\max }$ values $\leqslant 0.6$ could be either a small extracerebral hematoma or an intracerebral hematoma. Subdural hematomas, which layer out

Table 1 Summary of the initial NIRS findings in 305 patients with traumatic brain injury.

\begin{tabular}{lcc}
\hline & & \\
Type of injury & Number of patients & $\begin{array}{c}\Delta O D_{\max } \\
\text { median (range) }\end{array}$ \\
\hline $\begin{array}{l}\text { Diffuse brain injury } \\
\begin{array}{l}\text { Intracranial hematoma } \\
\text { Epidural hematoma }\end{array}\end{array}$ & $0.03(0-0.05)$ \\
Subdural hematoma & 49 & $1.12(0.12-1.60)^{*}$ \\
Intracerebral hematoma & 102 & $0.85(0.12-1.82)^{*}$ \\
$p$ value & 40 & $0.30(0.03-0.75)^{*}$ \\
\hline
\end{tabular}

Note: The $P$ value is for the Kruskall-Wallis test, and an asterisk identifies the injury types with $\triangle O D$ values that are significantly different by Dunn's method from the patients with diffuse brain injury. 
SUBDURAL HEMATOMAS

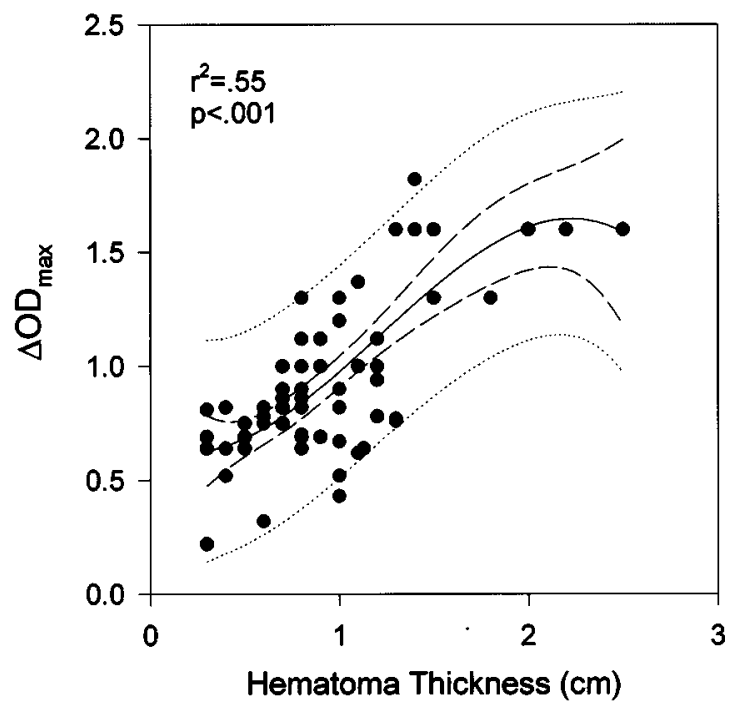

EPIDURAL HEMATOMAS

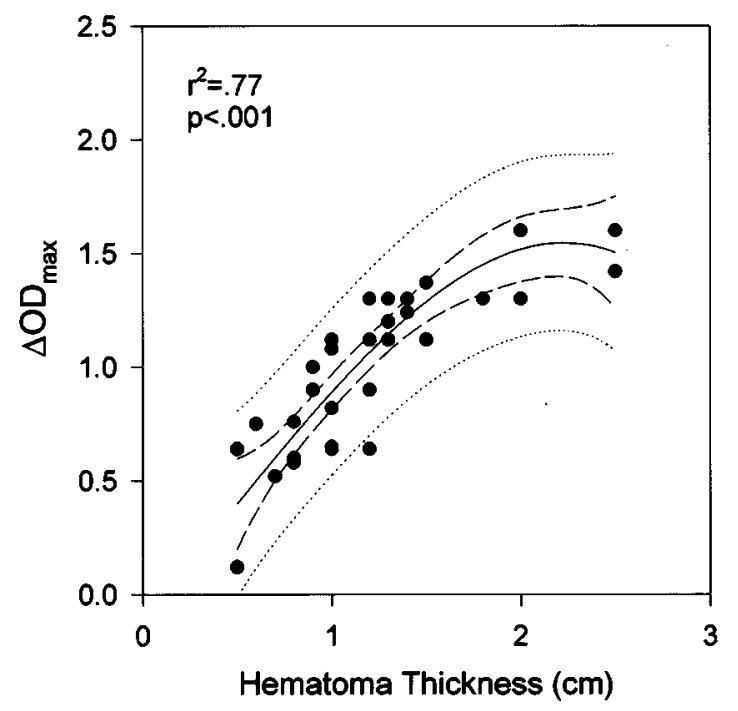

Fig. 2 Subdural hematomas (SDH) tend to layer out over the entire involved hemisphere, involving frontal, temporal, and parietal regions (left), while epidural hematomas (EDH) are confined by dural attachments to the sutures of the skull and tend to involve only one or two areas of the brain (middle). Intracerebral hematomas $(\mathrm{ICH})$ are confined by the surrounding brain parenchyma and tend to be localized to one or two areas of the brain (right). The $\triangle O D$ in the involved areas are all significantly different from the $\triangle O D$ in the corresponding brain region of the patients with diffuse brain injury (DBI).

over the cerebral hemisphere, tended to attenuate light in the frontal, parietal, and temporal regions of the involved hemisphere. Epidural hematomas, which are more localized hematomas confined by the attachment of the dura to the skull at sutures, tended to attenuate light in only one or two examination areas. Intracerebral hematomas, which are confined by the surrounding brain parenchyma, also usually altered reflected light intensity in only one or two examination areas. These differences in NIRS patterns with the different types of hematomas are illustrated in Figure 1, where the $\triangle O D$ s in the four areas of the brain examined are graphed.

\subsubsection{Patients with Multiple Intracranial Hematomas}

Most of the patients had a single intracranial lesion or multiple lesions in the same area of the brain, but 16 patients had bilateral hematomas. Because the NIRS examination relies on absorbance in the contralateral brain for comparison, bilateral lesions could be difficult to identify with this technology. This circumstance occurred on only two occasions in the 305 patients but must be kept in mind. In the other 14 patients, the second lesion occurred in another area of the brain, or occurred in a different time frame, and the NIRS examination clearly identified the presence of multiple hematomas (Figure 3).

\subsubsection{Patients with Deep Intracerebral Hematomas}

The depth that NIR light penetrates into the adult brain is controversial, but clearly deep intraparenchymal hematomas cannot be identified. After head trauma, most intracerebral hematomas and contusions are superficial, involving the frontal and temporal lobes of the brain. Figure 4 illustrates the only case out of the 305 patients where a basal ganglia contusion occurred. The maximal $\triangle O D$ in this patient was 0.03 , which was not significantly different from the patients with diffuse brain injury.

\subsection{NIRS MONITORING FOR DELAYED INTRACRANIAL HEMATOMAS IN THE INTENSIVE CARE UNIT}

The ability of NIRS to identify delayed intracranial hematomas in the intensive care unit was evaluated in the 305 patients. Fifty-nine (19\%) of the patients developed a late hematoma. In $33(11 \%)$ of the patients, the late hematoma was sufficiently large to require surgical evacuation.

To evaluate the performance of the NIRS, the $\triangle O D$ for the involved brain region was plotted against the clinical parameter that was being used to monitor the patient's neurological status-GCS for patients with mild or moderate head injury and ICP for patients with severe head injury. An increase in $\triangle O D$ to $>0.10$ was considered abnormal and to $>0.30$ was considered to be consistent with the presence of an intracranial hematoma of a size that might be a surgical lesion. A decrease in GCS 


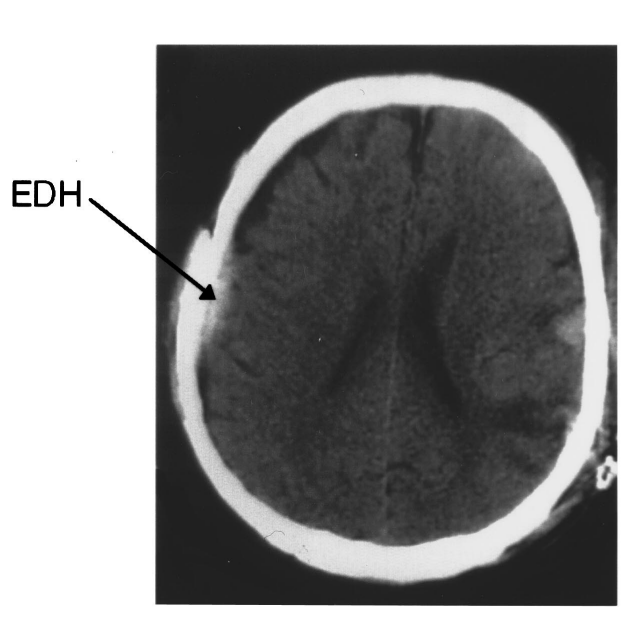

ER
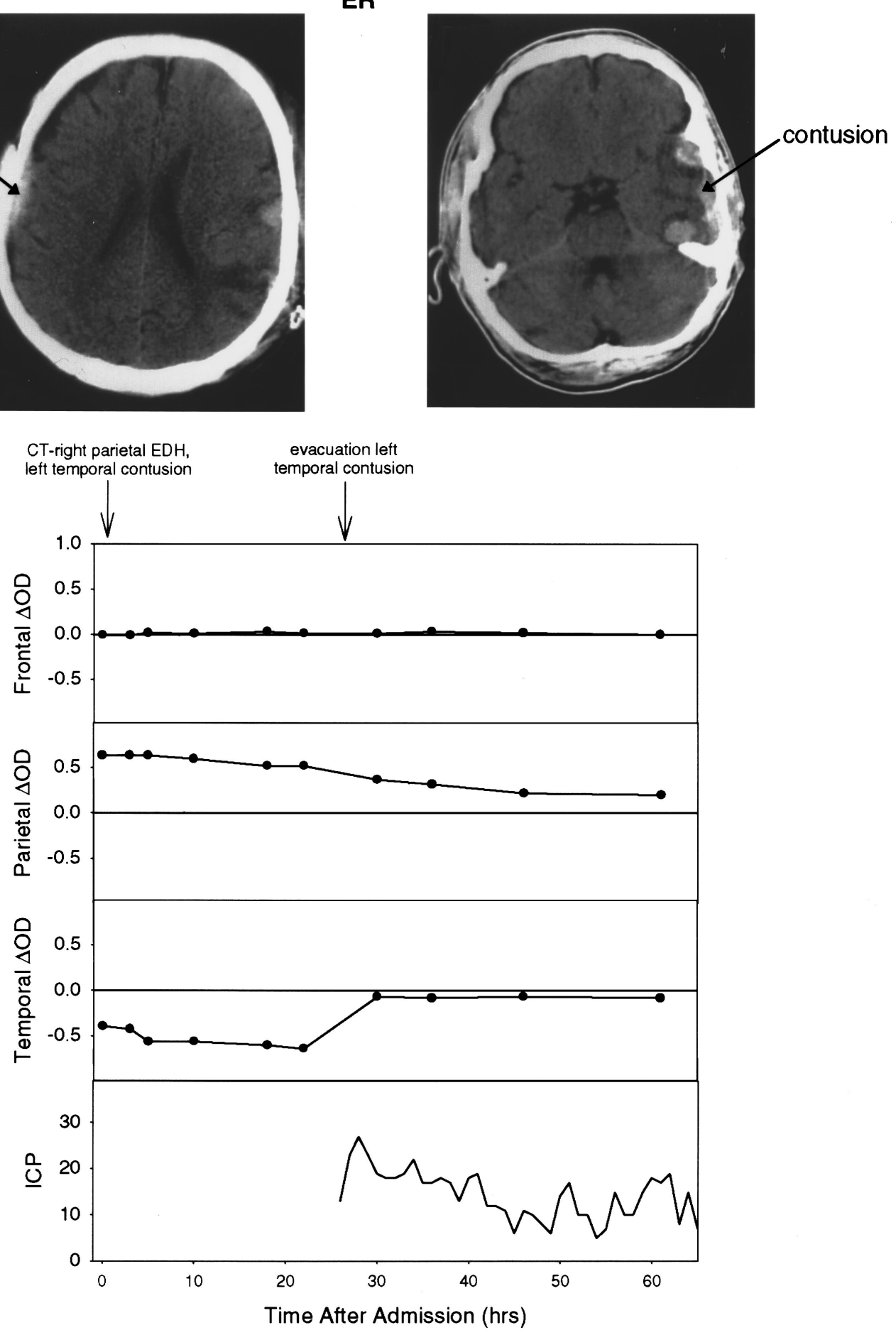

Fig. 3 This patient was admitted with two lesions, an epidural hematoma in the right parietal area, and a left temporal contusion. Because the hematomas occurred in two different areas in the brain, the NIRS examination identified both abnormalities. On day 2 after injury, he was taken to the operating room for evacuation of the temporal contusion. The epidural hematoma on the right resolved spontaneously.

of at least 2 points or an increase in ICP to at least $30 \mathrm{~mm} \mathrm{Hg}$ were considered to be abnormalities that should have indicated the possibility of a late hematoma. The CT scan that identified the late hematoma was used to mark the time that the routine clinical monitors (ICP or neurological examination) had actually indicated the possibility of development of a late hematoma to the clinicians caring for the patient. The monitor that provided the first indication of the development of the late hematoma was recorded. The 7 patients with diffuse brain injury who developed a delayed hematoma are 

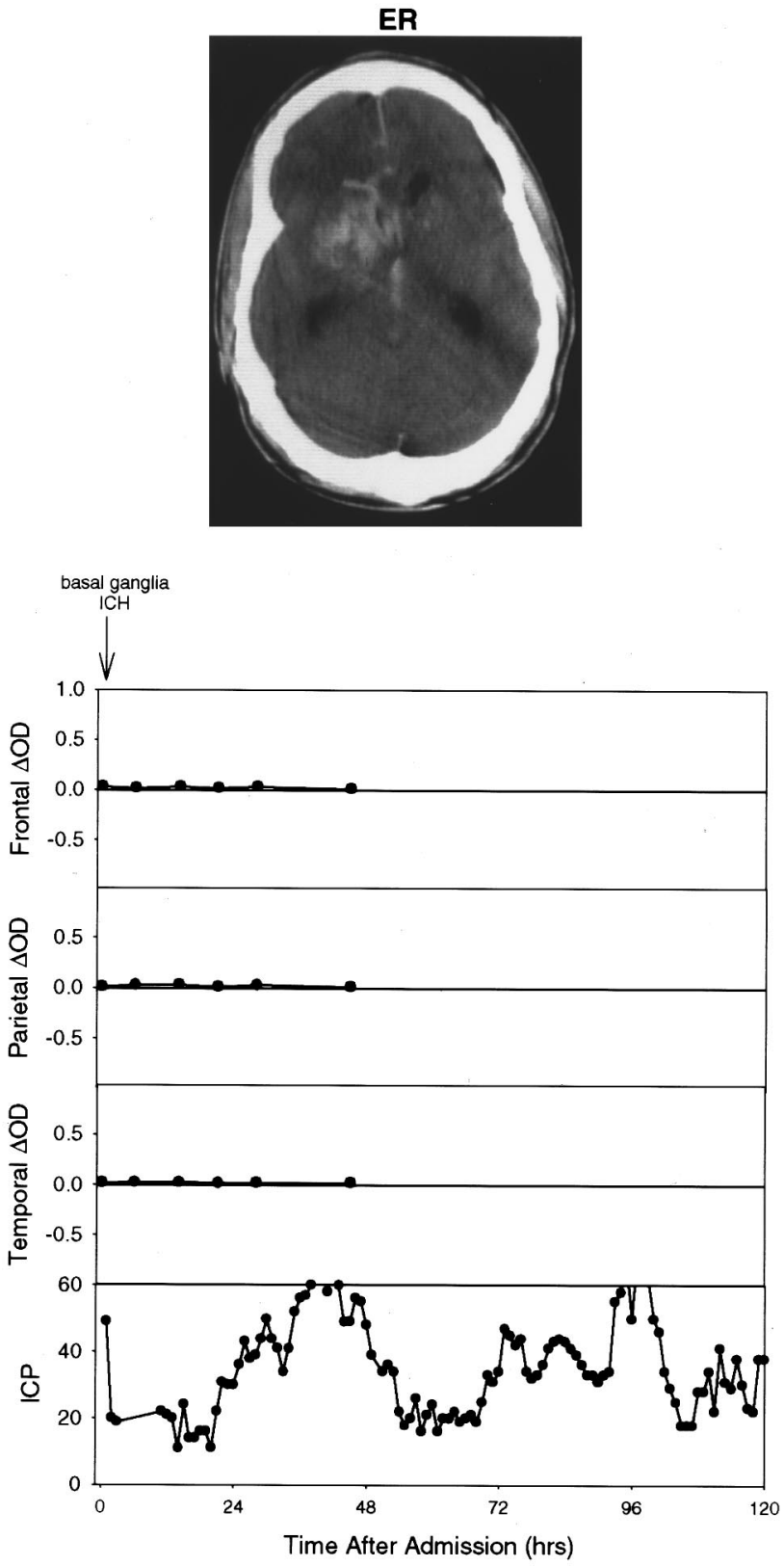

Fig. 4 This patient was admitted with a large basal ganglia contusion. Despite the large size of the lesion, there were no changes in $\triangle O D$, probably because the NIR light does not penetrate deep enough into the brain to be affected by the lesion.

shown as an example of these comparisons in Figure 5, and the data for all types of injury are summarized in Table 2 and Table 3.

\subsubsection{Initial Diagnosis of Diffuse Brain Injury}

One hundred fourteen patients had a diagnosis of diffuse brain injury on the initial CT scan obtained in the emergency room. Despite the absence of a hematoma on the initial evaluation, $7(6 \%)$ of the 114 patients developed a delayed hematoma during their hospital course. The delayed lesion was a contusion or an intracerebral hematoma in 6 of the patients. One patient developed a late epidural he- matoma. Four of these delayed hematomas required surgical evacuation. Three were treated medically.

The serial NIRS measurements of $\triangle O D$ in the involved area of the brain for these 7 patients are illustrated in Figure 5 and summarized in Table 2. The median $\triangle O D$ was 0.03 (range 0 to 0.05 ) on admission in all of the 7 patients who subsequently developed late hematomas. Within 2 to $24 \mathrm{~h}$ after admission, the $\triangle O D$ began to increase, and was significantly higher than the patients without late hematomas at the time of recognition of the hematoma development (Table 2). Postoperatively, in the 4 patients who had surgical evacuation of the hematoma, the $\triangle O D$ returned to normal. In the patients who were treated medically, the $\triangle O D$ gradually returned to normal as the hematoma resolved.

\subsubsection{Initial Diagnosis of Epidural Hematoma}

Forty-nine patients were admitted with an epidural hematoma. Thirty of these hematomas required surgical evacuation, and 19 were small and were treated medically. Six $(20 \%)$ of the 30 surgical patients developed a late hematoma. These included 2 recurrent epidural hematomas, 1 new epidural hematoma, and 3 delayed traumatic intracerebral hematomas. Three of these 6 late hematomas required surgical evacuation. Three $(16 \%)$ of the 19 patients who were initially treated medically had enlargement of the epidural hematoma and two subsequently required surgical evacuation of the epidural hematoma.

The distribution of the $\Delta O D_{\max }$ on admission to the hospital for the group of patients with epidural hematomas is summarized in Table 1. The median $\Delta O D_{\max }$ of the patients requiring surgery was significantly higher $(P<0.001)$ than that of the patients treated medically, 1.30 (range 0.64 to 1.60 ) compared to 0.62 (range 0.12 to 1.08 ), respectively. This difference probably reflects the larger size of the hematoma in the patients requiring surgery.

The serial measurements of $\Delta O D_{\max }$ are summarized in Table 2. In the surgical patients, the $\Delta O D_{\max }$ returned to normal in the 23 with uncomplicated postoperative courses. The $\Delta O D_{\max }$ increased again postoperatively to a median value of 0.46 (range 0.14 to 1.00 ) in the 6 patients who developed a late hematoma after surgery. In the medical patients, the $\triangle O D_{\max }$ gradually decreased toward normal in the 15 patients in whom the epidural hematoma spontaneously resolved. In contrast, the maximal $\triangle O D$ increased to a median value of 0.64 (range 0.52 to 1.12) in the 3 patients in whom the epidural hematoma enlarged and subsequently required surgical evacuation.

\subsubsection{Initial Diagnosis of Subdural Hematoma}

One hundred and two patients were admitted with a subdural hematoma. Eighty-four of these were large enough to require surgical evacuation on ad- 


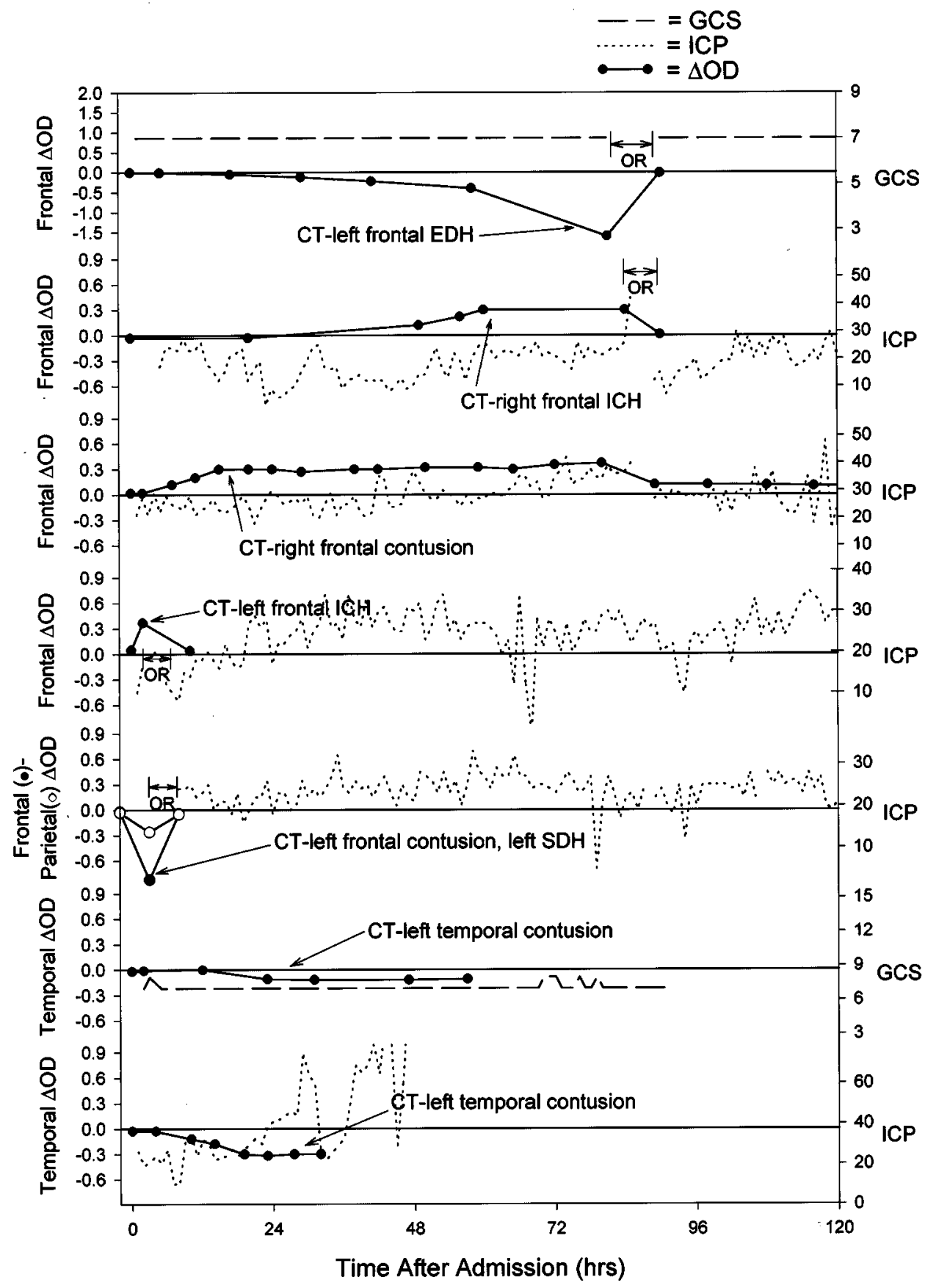

Fig. 5 The serial changes in NIRS light intensity in the involved region of the brain and the changes in intracranial pressure or Glasgow coma score in the 7 patients who had an initial diagnosis of diffuse brain injury and developed a late hematoma. $\mathrm{CT}$, computerized tomography, $\mathrm{OR}$, operating room; EDH, epidural hematoma, ICH, intracerebral hematoma; SDH, subdural hematoma; ICP, intracranial pressure, GCS, Glasgow coma score.

mission to the hospital. Eighteen were small and were treated medically. Of the entire group of 102 patients with a subdural hematoma, 24 (24\%) developed a late hematoma. These included 13 recurrent subdural hematomas, 4 postoperative epidural hematomas, 1 new epidural hematoma, 1 new subdural hematoma, and 5 intracerebral hematomas. Ten of the 24 late hematomas were surgical lesions.
The distribution of the $\Delta O D_{\max }$ on admission to the hospital for the group of subdural hematomas is summarized in Table 1 . The median $\triangle O D_{\max }$ of the patients requiring surgery was significantly higher $(P<0.001)$ than that of the patients treated medically, 0.90 (range 0.32 to 1.82 ) compared to 0.64 (range 0.12 to 1.12), respectively. As with the epidural hematomas, this difference probably reflects the 
Table 2 Comparison of $\Delta O D_{\max }$ values in patients who developed a late intracranial hematoma and patients who had uncomplicated courses.

\begin{tabular}{|c|c|c|c|c|c|}
\hline \multirow{2}{*}{$\begin{array}{l}\text { Initial diagnosis } \\
\text { and } \\
\text { initial treatment }\end{array}$} & \multirow[b]{2}{*}{$\begin{array}{c}\text { No. with late } \\
\text { hematomas }\end{array}$} & \multicolumn{3}{|c|}{$\Delta O D_{\max }$} & \multirow{2}{*}{$\begin{array}{c}P \text { Value } \\
\text { (Comparison o } \\
\Delta O D \text { in the } 2 \\
\text { groups) }\end{array}$} \\
\hline & & $\begin{array}{c}\text { Patients without late } \\
\text { hematomas }\end{array}$ & $\begin{array}{r}\text { Patie } \\
\mathrm{h}\end{array}$ & $\begin{array}{l}\text { nts with late } \\
\text { matomas }\end{array}$ & \\
\hline $\mathrm{DBI}$ & $7 / 114(6 \%)$ & $0.03(0-0.05)$ & 0.55 & $(0.12-1.60)$ & $<0.001$ \\
\hline \multicolumn{6}{|l|}{$\mathrm{EDH}$} \\
\hline Surgical treatment & $6 / 30(20 \%)$ & $0.02(0-0.03)$ & 0.46 & $(0.14-1.00)$ & $<0.001$ \\
\hline Medical treatment & $3 / 19(16 \%)$ & $0.12(0.03-0.30)$ & 0.64 & $(0.52-1.12)$ & $<0.001$ \\
\hline \multicolumn{6}{|l|}{ SDH } \\
\hline Surgical treatment & $24 / 84(29 \%)$ & $0.03(0.01-0.06)$ & 0.53 & $(0.11-1.20)$ & $<0.001$ \\
\hline Medical treatment & $0 / 18 \quad(0 \%)$ & $0.09(0.02-0.15)$ & & & \\
\hline \multicolumn{6}{|l|}{$\mathrm{ICH}$} \\
\hline Surgical treatment & $5 / 18(28 \%)$ & $0.03(0.01-0.05)$ & 0.64 & $(0.16-1.00)$ & 0.002 \\
\hline Medical treatment & $14 / 22(64 \%)$ & $0.11(0.03-0.12)$ & 0.43 & $(0.20-0.82)$ & $<0.001$ \\
\hline
\end{tabular}

Note: Regardless of the type of hematoma and subsequent initial treatment, the patients who developed intracranial hematomas had a significantly higher $\Delta O D_{\max }$

larger size of the hematomas in the surgical group.

The changes in the $\Delta O D_{\max }$ with treatment are summarized in Table 2. In the 60 surgical patients with an uncomplicated postoperative course, the $\triangle O D$ returned to normal. In the 24 surgical patients who developed a late hematoma, the $\triangle O D$ increased again postoperatively to a median value of 0.53 (range 0.11 to 1.20 ). In the 18 medical patients, the $\triangle O D$ gradually returned toward normal over several days as the subdural blood was reabsorbed.

\subsubsection{Initial Diagnosis of Contusion or Intracerebral Hematoma}

Forty patients were admitted with a contusion or intracerebral hematoma. Eighteen of these were large enough to require surgical evacuation. Twenty-two were initially treated medically. Five of the 18 surgical patients developed a late he- matoma. Two were recurrent intracerebral hematomas, 2 were postoperative epidural hematomas, and 1 was a new subdural hematoma. Four of these late hematomas required surgical evacuation, and 1 was treated medically. Fifteen of the patients who were initially treated medically had enlargement of their hematoma, with 10 requiring surgical evacuation.

The distribution of the $\triangle O D_{\max }$ for the group of intracerebral hematomas is summarized in Table 1. The median $\triangle O D$ of the patients requiring surgery was significantly higher $(P=0.001)$ than that of the patients who were treated medically, 0.39 (range 0.09 to 0.75 ) compared to 0.15 (range 0.03 to 0.49 ), respectively.

The changes in the $\Delta O D_{\max }$ with treatment are summarized in Table 2 . In the 13 surgical patients with an uncomplicated postoperative course, the

Table 3 Comparison of NIRS and routine clinical monitors as early detectors of late intracranial hematoma.

\begin{tabular}{lccccc}
\hline & \multicolumn{5}{c}{ Earliest indicator of late hematoma } \\
\cline { 2 - 5 } & $\begin{array}{c}\text { NIRS } \\
(\Delta O D>0.10)\end{array}$ & $\begin{array}{c}\text { ICP } \\
(>30 \mathrm{~mm} \mathrm{Hg})\end{array}$ & $\begin{array}{c}\text { GCS } \\
(\downarrow 2 \text { points })\end{array}$ & $\begin{array}{c}\text { Routine } \\
\text { follow-up } \\
\text { CT scan }\end{array}$ & $\begin{array}{c}\text { Number with } \\
\Delta O D>0.3\end{array}$ \\
\begin{tabular}{l} 
Type of late hematoma \\
\hline $\begin{array}{l}\text { Large hematoma, } \\
\text { requiring surgery }\end{array}$
\end{tabular} & 30 & 3 & 0 & 0 & $31 / 33(94 \%)$ \\
$\begin{array}{l}\text { Small hematoma, treated } \\
\text { medically }\end{array}$ & 25 & 0 & 0 & 1 & $17 / 26(65 \%)$ \\
\hline
\end{tabular}


$\triangle O D$ returned to normal. In the 5 surgical patients who developed a late hematoma, the $\triangle O D$ remained elevated postoperatively, a median value of 0.64 (range 0.16 to 1.00 ). In the 15 medical patients who developed late enlargement of the hematoma, the $\triangle O D$ increased to 0.43 (range 0.20 to 0.82 ) while in the 7 patients in whom the hematoma resolved spontaneously, the $\triangle O D$ gradually returned toward normal over several days as the blood was reabsorbed.

\subsubsection{Relationship of the Changes in $\triangle O D$ to Other Clinical Monitors}

The clinical signs that are routinely used in the intensive care unit to indicate the development of an intracranial hematoma are increasing ICP (to $>30$ $\mathrm{mm} \mathrm{Hg}$ ) and/or deteriorating neurological examination (decrease in the GCS of at least 2 points or signs of tentorial herniation). Table 3 compares the ability of these routine monitors and the NIRS monitor to identify intracranial hematomas. For the entire group of 59 patients with late hematomas, the NIRS detected the presence of the hematoma earlier than the ICP or the neurological examination in 55 (93\%). In 2 patients, the ICP increased prior to the change in $\Delta O D_{\max }$. In two patients who developed bilateral hematomas, the $\Delta O D_{\max }$ did not change significantly and the delayed lesion was identified by a rising ICP in one patient and on a routine follow-up CT scan in the other patient. The latter patient is illustrated in Figure 3.

Since late hematomas are most important to identify if they are large enough to require surgical evacuation, the performance of the NIRS monitor in the 33 patients requiring surgery was compared with the 26 patients who were treated medically for the late hematoma. The $\Delta O D_{\max }$ increased to at least 0.3 in $31 / 33$ of the patients with a surgical late hematoma, compared with only $17 / 26$ in the patients with a late hematoma that was small enough that it could be managed medically. The 2 surgical hematomas that had a $\triangle O D<0.3$ were frontal intracerebral hematomas.

\section{DISCUSSION}

The NIRS measurements as described in this study were highly specific for intracranial hematomas. There were no cases identified in which the $\Delta O D_{\max }$ was $>0.1$ but an intracranial hematoma could not be identified on a CT scan in the corresponding region of the brain. In contrast, ICP and neurological examination are very nonspecific. Intracranial hypertension occurs after trauma from cerebral edema and hyperemia much more commonly than from an intracranial hematoma.

Scalp hematomas are a potential problem in trauma patients that could confound the NIRS measurements. Recent studies have documented that in the adult the contribution of extracerebral tissues to the NIRS signal is dependent upon the separation of the light source and sensor, and also on the area of skull monitored.

With optode separations of less than $4 \mathrm{~cm}$, extracerebral tissues contribute the majority of the NIRS signal. At optode separations up to $7 \mathrm{~cm}$, extracerebral contamination is still present. ${ }^{17}$ Studies using selective injection of indocyanine green into the internal and external carotid arteries showed that the internal carotid signal was negligible at an optode separation of $1.0 \mathrm{~cm} .{ }^{18}$ Below optode separations of $3 \mathrm{~cm}$, the contribution of the internal carotid signal was proportional to the separation distance. Between optode separations of 3 and $7 \mathrm{~cm}$, the external carotid signal was constant.

Each region of the skull has variable thicknesses of the tissue layers that may influence the NIRS signal. The temporal area, in particular, has a significant amount of muscle mass which may contribute to the NIRS signal. Teeth clenching, for example, has been shown to alter the NIRS signal when the probe is placed over the temporalis muscle. ${ }^{19}$ Rapid frontalis muscle exercise alters the NIRS signal when the probe is placed on the forehead. ${ }^{20}$

In practice, scalp hematomas can be easily identified and avoided in most patients. Patients with massive scalp trauma cannot be monitored with this technique and were excluded from the present study. The difference in the extracerebral tissues at each of the four brain regions monitored was minimized by comparing the NIRS signal of each area with the same area of the brain on the opposite side. Using this approach, there was a very low variability for $\triangle O D$ in the different brain regions of patients without intracranial hematomas.

The NIRS measurements were also more sensitive than the monitors that are currently being used to follow patients with head trauma. The $\triangle O D$ increased in the presence of a developing intracranial hematoma prior to changes in intracranial pressure or neurological examination in all but 4 cases.

However, there are several limitations for identifying intracranial hematomas with NIRS that were observed in this study. First, the size, type, and location of the hematoma cannot be as precisely determined as with a CT scan. Intracerebral hematomas tend to absorb light less intensely than extracerebral hematomas, and a $\Delta O D>0.6$ strongly suggests an extracerebral hematoma, but there is considerable overlap when the $\triangle O D$ is $\leqslant 0.6$. Subdural hematomas tend to involve all examination areas in the involved hemisphere, while epidural and intracerebral hematomas tend to have more localized changes. Clearly, a CT scan is necessary to obtain the detailed information necessary to make surgical decisions. However, the NIRS examination may have a role in identifying patients in the intensive care unit who require repeat CT scans.

Second, because the NIRS examination relied on a comparison of reflected light from normal brain to 
ER

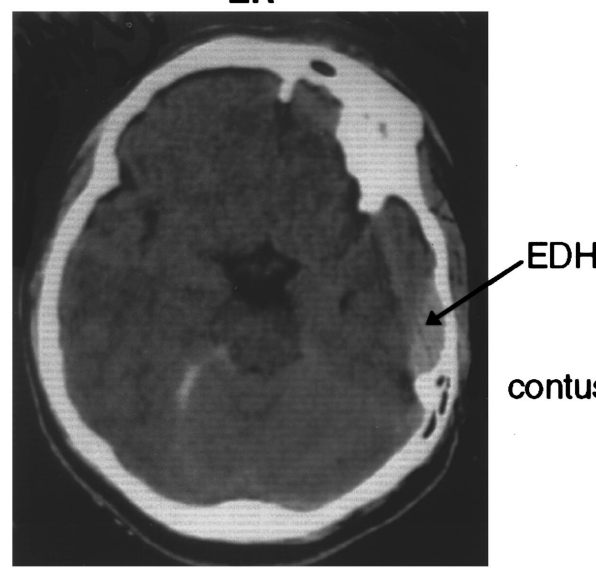

CT-increase in EDH +

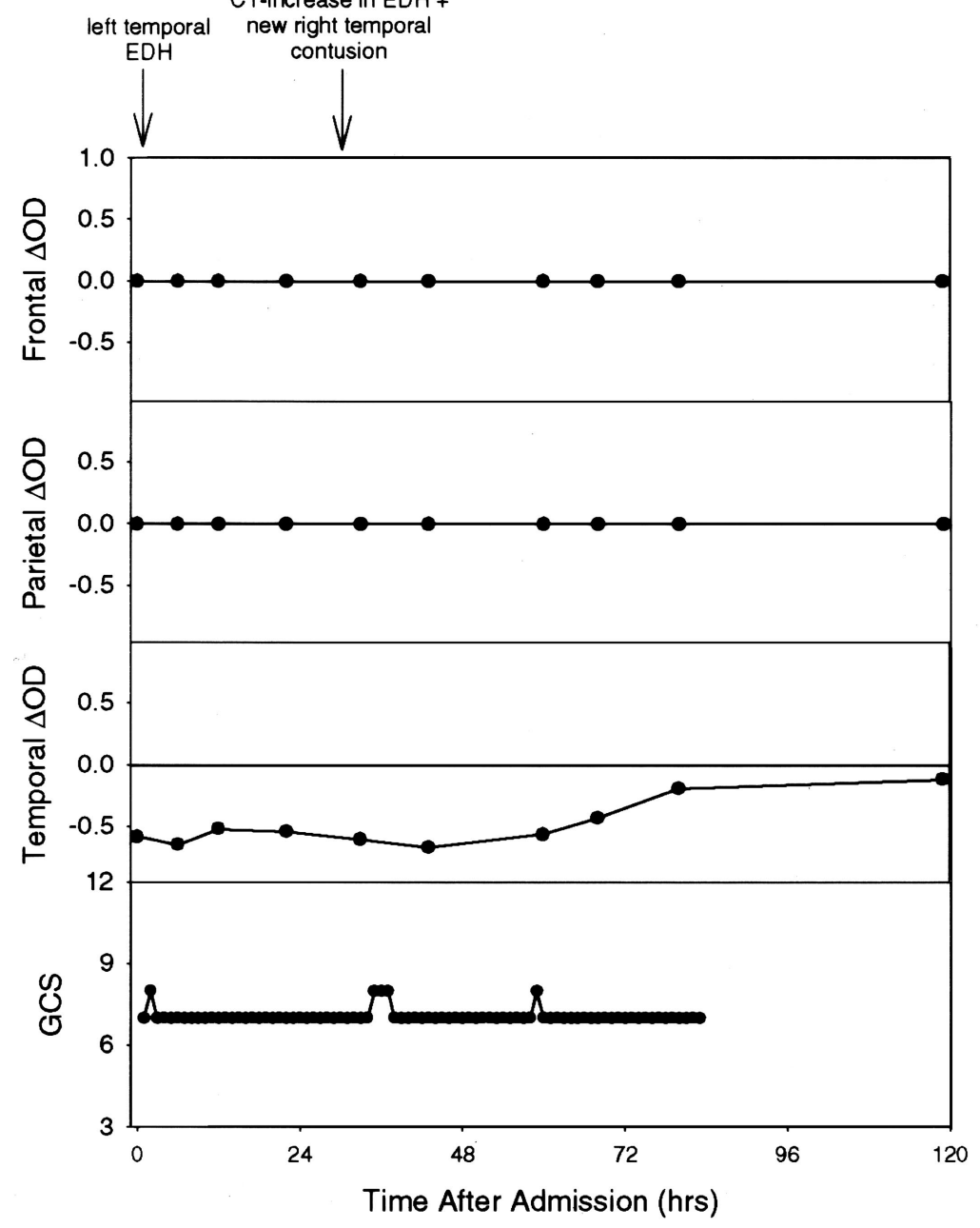

Day 2

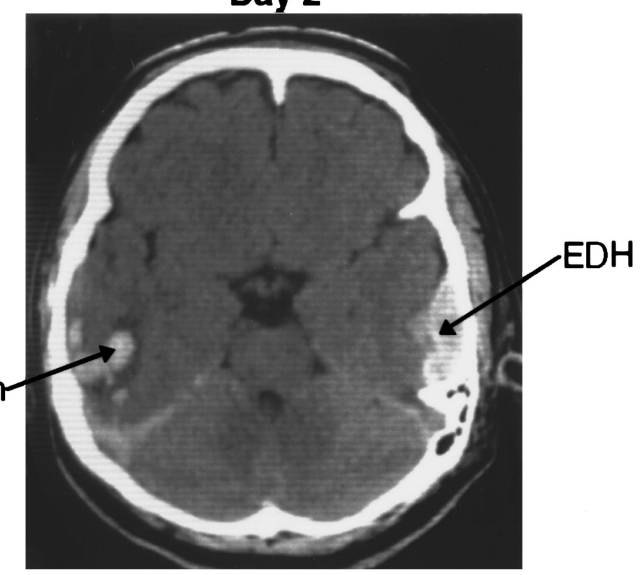


Third, while the exact depth of brain examined by NIRS light is controversial, it is clear that deep intraparenchymal hematomas cannot be detected with the NIRS probe used in the present study. Fortunately, this type of intracerebral hematoma is not common after trauma, occurring only once in the 305 patients in the present study. However, for other types of intracranial hemorrhages which more commonly involve the deep brain structures, the intracranial blood would be more difficult to detect with NIRS.

\section{Acknowledgments}

This work was supported by Texas Advanced Technology Program Grant \#004949-022 and by National Institutes of Health Grants \#PO1-NS27616 and NS27346.

\section{REFERENCES}

1. Y. Sawada, D. Sadamitsu, T. Sakamoto, K. Ikemura, T. Yoshioka, and T. Sugimoto, "Lack of correlation between delayed traumatic intracerebral hematoma and disseminated intravascular coagulation," J. Neurol. Neurosurg. Psych. 47 (10), 1125-1127 (1984).

2. S. K. Gudeman, P. R. S. Kishore, J. D. Miller, A. K. Girevendulis, J. H. Lipper, and D. P. Becker, "The genesis and significance of delayed traumatic intracerebral hematoma," Neurosurgery 5 (3), 309-313 (1979).

3. M. H. Lipper, P. R. Kishore, A. K. Girevendulis, J. D. Miller, and D. P. Becker, "Delayed intracranial hematoma in patients with head injury," Radiology 133 (3), 645-649 (1979).

4. R. C. Sweet, J. D. Miller, and M. H. Lipper, "Significance of bilateral abnormalities on the CT scan in patients with severe head injury," Neurosurgery 3 (1), 16-21 (1978).

5. H. A. Young, G. R. W. Gleave, H. H. Schmidek, and S. Gregory, "Delayed traumatic intracerebral hematoma: report of 15 cases operatively treated," Neurosurgery 14 (1), 22-25 (1984).

6. A. Fukamachi, Y. Nagaseki, K. Kohno, and T. Wakao, "The incidence and developmental process of delayed traumatic intracerebral hematomas," Acta Neurochir. 74 (1), 35-39 (1985).

7. D. Gentleman, F. Nath, and P. MacPherson, "Diagnosis and management of delayed traumatic intracerebral hematomas," Br. J. Neurosurg. 3 (3), 367-372 (1989).
8. G. L. Clifton, R. G. Grossman, M. E. Makela, M. E. Miner, S. Handel, and V. Sadhu, "Neurological course and correlated computerized tomography findings after severe closed head injury,'" J. Neurosurg. 52 (5), 611-624 (1980).

9. B. Borovich, J. Braun, J. N. Guilburd, M. Zaaroor, M Michich, L. Levy, A. Lemberger, I. Grushkiewicz, M. Feinsod, and I. Schachter, "Delayed onset of traumatic extradural hematoma," J. Neurosurg. 63 (1), 30-34 (1985).

10. J. M. Piepmeier and F. C. Wagner, Jr., "Delayed posttraumatic extracerebral hematomas," J. Trauma 22 (6), 455460 (1982).

11. T. Ninchoji, K. Nemura, I. Shimoyama, K. Hinokuma, T. Bun, and S. Najakima, "Traumatic intracerebral haematomas of delayed onset," Acta Neurochir. 71 (1), 69-90 (1984).

12. F. G. Diaz, D. H. Yock, D. Larson, and G. L. Rockswold "Early diagnosis of delayed posttraumatic intracerebral hematomas," J. Neurosurg. 50 (2), 217-223 (1979).

13. H. Sakai, H. Takagi, H. Ohtaka, T. Tanabe, T. Ohwada, and K. Yada, "Changes of Glasgow coma score and ICP during the development of acute epidural hematoma," in Intracranial Pressure, Vol. VI, J. D. Miller, G. M. Teasdale, J. O. Rowan, S. L. Galbraith, and A. D. Mendelow, Eds., pp. 652 655, Springer-Verlag, Berlin (1986).

14. B. T. Andrews, L. H. Pitts, M. P. Lovely, and H. Bartowski, "Is computed tomographic scanning necessary in patients with tentorial herniation? Results of immediate surgical exploration without computed tomography in 100 patients," Neurosurgery 19 (3), 408-414 (1986).

15. R. Bullock, J. Golek, and G. Blake, "Traumatic intracerebral hematoma-which patients should undergo surgical evacuation? CT scan features and ICP monitoring as a basis for decision making," Surg. Neurol. 32 (3), 181-187 (1989).

16. S. P. Gopinath, C. S. Robertson, R. G. Grossman, and B. Chance, "Near-infrared spectroscopic localization of intracranial hematomas," J. Neurosurg. 79 (1), 43-47 (1993).

17. D. N. Harris, F. M. Cowans, D. A. Wertheim, and S. Hamid, "NIRs in adults-effects of increasing optode separation," Adv. Exp. Med. Biol. 345, 837-840 (1994).

18. P. W. McCormick, M. Stewart, G. Lewis, M. Dujovny, and J. L. Ausman, "Intracerebral penetration of infra-red light," $J$. Neurosurg. 76 (2), 315-319 (1992).

19. D. N. Harris, F. M. Cowans, and D. A. Wertheim, "NIRS in the temporal region-strong influence of external carotid artery," Adv. Exp. Med. Biol. 345, 825-828 (1994).

20. T. J. Germon, N. M. Kane, A. R. Manara, and R. J. Nelson, "Near-infrared spectroscopy in adults: effects of extracranial ischemia and intracranial hypoxia on estimation of cerebral oxygenation," Br. J. Anaesthesia 73 (4), 503-506 (1994). 